\title{
A Bound on the Implicit Degree of Polygonal Bézier Surfaces
}

\author{
Joe Warren \\ Department of Computer Science \\ Rice University *
}

October 1990

\begin{abstract}
Recent work has shown that the triangular rational Bézier surface representation can be used to create polygonal surface patches. The key to the construction is the judicious use of zero weights in creating the surface parameterization. These zero weights introduce base points into the resulting rational parameterizations. Base points also lower the degree of the implicit representation of these polygonal surface patches. This paper states and proves in a simple, constructive manner a bound on the implicit degree of rational surfaces created in this manner.
\end{abstract}

\section{Introduction}

Triangular parametric surface patches are an important tool in geometric modeling. Mathematically, a triangular surface patch $S$ is the image of a domain triangle $T$ under some parametric mapping. If $T$ has vertices $\mathbf{v}_{0}, \mathbf{v}_{1}$, and $\mathbf{v}_{2}$, any point $\mathbf{q}$ in $T$ can be expressed uniquely in the form $\mathbf{q}=\alpha_{0} \mathbf{v}_{0}+\alpha_{1} \mathbf{v}_{1}+\alpha_{2} \mathbf{v}_{2}$ where $\alpha_{0}, \alpha_{1}, \alpha_{2} \geq 0$ and $\sum \alpha_{i}=1$; in this case, $\left(\alpha_{0}, \alpha_{1}, \alpha_{2}\right)$ are called the barycentric coordinates of $\mathbf{q}$ with respect to the domain triangle $T$.

A rational Bézier surface of parametric degree $d$ is of the form:

$$
\left(\begin{array}{l}
x \\
y \\
z \\
w
\end{array}\right)=\sum_{\substack{i_{0}, i_{1}, i_{2} \geq 0 \\
i_{0}+i_{1}+i_{2}=d}}\left(\begin{array}{c}
x_{i_{0} i_{1} i_{2}} w_{i_{0} i_{1} i_{2}} \\
y_{i_{0} i_{1} i_{2}} w_{i_{0} i_{1} i_{2}} \\
z_{i_{0} i_{1} i_{2}} w_{i_{0} i_{1} i_{2}} \\
w_{i_{0} i_{1} i_{2}}
\end{array}\right) B_{i_{0} i_{1} i_{2}}\left(\alpha_{0}, \alpha_{1}, \alpha_{2}\right)
$$

* Supported in part by NSF grant CCR 89-03431 
where $B_{i_{0} i_{1} i_{2}}$ are the bivariate Bernstein basis functions of degree $d$. These basis functions have the form:

$$
B_{i_{0} i_{1} i_{2}}\left(\alpha_{0}, \alpha_{1}, \alpha_{2}\right)=\frac{d !}{i_{0} ! i_{1} ! i_{2} !} \alpha_{0}^{i_{0}} \alpha_{1}^{i_{1}} \alpha_{2}^{i_{2}} .
$$

The parameterization yields points whose homogeneous coordinates are $(x, y, z, w)$. Since the affine coordinates of this point are of the form $\left(\frac{x}{w}, \frac{y}{w}, \frac{z}{w}\right)$, the resulting affine surface patch is the image of a rational function. This representation is useful in geometric design because coefficients of these functions have geometric meaning. The points $\left(x_{i_{0} i_{1} i_{2}}, y_{i_{0} i_{1} i_{2}}, z_{i_{0} i_{1} i_{2}}\right)$ are called control points. Associated with each control point is a weight $w_{i_{0} i_{1} i_{2}}$. The set of all control points and their weights forms a weighted control polyhedron that approximates the resulting surface patch. [Far88] describes some of the properties of rational Bézier surfaces.

\section{Base points}

Parameter values for which $(x, y, z, w)=(0,0,0,0)$ are called base points of the parameterization. It is easy to introduce a base point into the parameterization of a rational Bézier surface. For example, when the weight $w_{d 00}$ is zero, the values of $x, y, z$, and $w$ are all zero at the parameter value $(1,0,0)$. Base points have two important effects on rational surfaces.

First, the image of a base point under the rational parameterization is a set of rational curves on the resulting rational surface [Cle68, $\left.\mathrm{S}^{+} 70\right]$. This observation follows from the fact that at a base point, the rational parameterization evaluates to $\left(\frac{0}{0}, \frac{0}{0}, \frac{0}{0}\right)$. The image of the base point depends on the direction in the parameter plane from which the base point is approached. This observation is the basis for a method to create polygonal patches [War92].

Let $S$ be a rational Bézier surface patch of parametric degree $d$ where $S$ is the image of a domain triangle $T$ with barycentric coordinates $\left(\alpha_{0}, \alpha_{1}, \alpha_{2}\right)$. The convex hull of the points $\left\{\left(i_{0} / d, i_{1} / d, i_{2} / d\right) \mid w_{i_{0} i_{1} i_{2}} \neq 0\right\}$ is known as the Newton polygon of the weight function of $S$ [Wa150]. The Newton polygon provides a key to understanding the structure of $S$. The following theorem from [War92] demonstrates its importance.

Theorem 1 Let $P$ be the Newton polygon of the weight function for a rational Bézier surface patch $S$. The boundary curves of $S$ are in $1-1$ correspondence with edges of $P$.

The control points of $S$ that lie on a single edge of $P$ define a rational Bézier curve that is a boundary curve of $S$. Figure 1 illustrates the relationship between $P$ and $S$ and shows 
a hexagonal surface patch created using this technique.

The other important effect of base points is that their presence lowers the implicit or algebraic degree of $S$. Any rational surface patch $S$ can be expressed implicitly as the zero set of a homogeneous polynomial function [Har77]

$$
F(x, y, z, w)=0
$$

The degree of the polynomial $F$ is called the implicit or algebraic degree of $S$. The following argument, from [Sal14, Sed90], illustrates the relationship between the degree of a rational parameterization, its number of base points, and the implicit degree of resulting surface patch.

The implicit degree of $S$ is equivalent to the number of intersections of $S$ and a line $L$ [Har77, Ch. I]. Define $L$ as the intersection of the planes with equations

$$
\begin{aligned}
& \lambda_{1} x+\lambda_{2} y+\lambda_{3} z+\lambda_{4} w=0 \\
& \delta_{1} x+\delta_{2} y+\delta_{3} z+\delta_{4} w=0
\end{aligned}
$$

If $\left(x\left(\alpha_{0}, \alpha_{1}, \alpha_{2}\right), y\left(\alpha_{0}, \alpha_{1}, \alpha_{2}\right), z\left(\alpha_{0}, \alpha_{1}, \alpha_{2}\right), w\left(\alpha_{0}, \alpha_{1}, \alpha_{2}\right)\right)$ is a parameterization for $S$, then each intersection of $S$ with one of the planes defining $L$ is a curve in the parameter space of
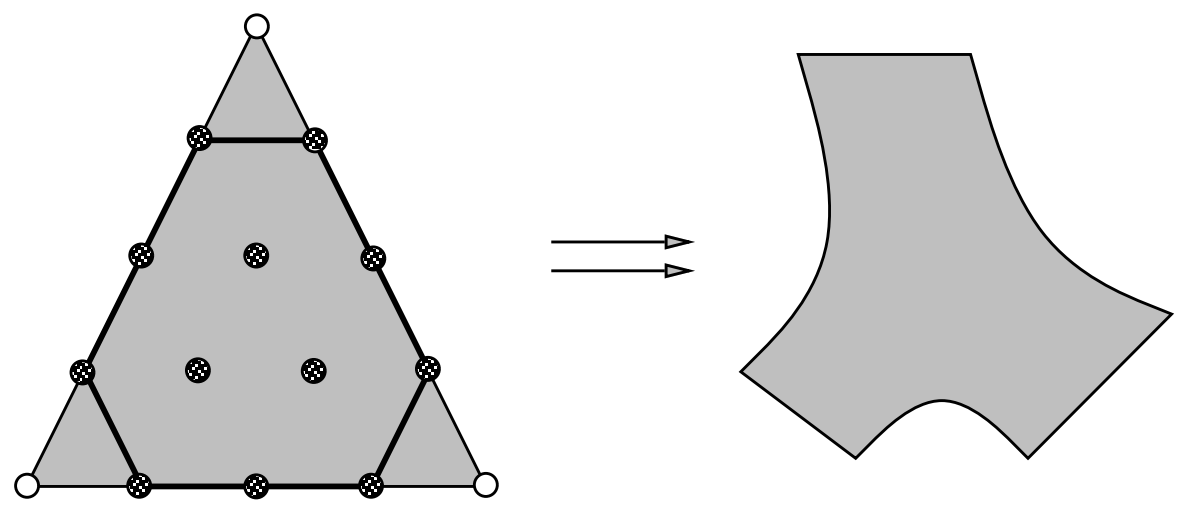

Zero weights

(2) Nonzero weights

Newton polygon

FigurE 1: Transforming a domain triangle into a hexagonal patch 
$S$. These curves are $f=0$ and $g=0$ where

$$
\begin{aligned}
& f\left(\alpha_{0}, \alpha_{1}, \alpha_{2}\right)=\lambda_{1} x\left(\alpha_{0}, \alpha_{1}, \alpha_{2}\right)+\lambda_{2} y\left(\alpha_{0}, \alpha_{1}, \alpha_{2}\right)+\lambda_{3} z\left(\alpha_{0}, \alpha_{1}, \alpha_{2}\right)+\lambda_{4} w\left(\alpha_{0}, \alpha_{1}, \alpha_{2}\right) \\
& g\left(\alpha_{0}, \alpha_{1}, \alpha_{2}\right)=\delta_{1} x\left(\alpha_{0}, \alpha_{1}, \alpha_{2}\right)+\delta_{2} y\left(\alpha_{0}, \alpha_{1}, \alpha_{2}\right)+\delta_{3} z\left(\alpha_{0}, \alpha_{1}, \alpha_{2}\right)+\delta_{4} w\left(\alpha_{0}, \alpha_{1}, \alpha_{2}\right)
\end{aligned}
$$

Bézoutś theorem [Ful78] guarantees that the curves $f=0$ and $g=0$ intersect in at most $d^{2}$ points in parameter space. However, not all of these points necessarily correspond to points of $L \cap S$. Base points of the parameterization account for some of these intersections in parameter space. Taking the $u$-resultant [vdW49] of

$$
\begin{aligned}
f\left(\alpha_{0}, \alpha_{1}, \alpha_{2}\right) & =0 \\
g\left(\alpha_{0}, \alpha_{1}, \alpha_{2}\right) & =0 \\
\alpha_{0} u_{0}+\alpha_{1} u_{1}+\alpha_{2} u_{2} & =0
\end{aligned}
$$

yields a homogeneous polynomial of degree $d^{2}$ in $u_{0}, u_{1}$, and $u_{2}$. This polynomial decomposes into linear factors of the form $p_{0} u_{0}+p_{1} u_{1}+p_{2} u_{2}$. In some of the factors, the coefficients $p_{i}$ may be constants, independent of the coefficients $\lambda_{i}$ and $\delta_{i}$. These points $\left(p_{0}, p_{1}, p_{2}\right)$ in parameter space correspond to intersections of $f=0$ and $g=0$ that are independent of the choice of the line $L$. If $L=(x=0) \cap(y=0)$, then $x\left(p_{0}, p_{1}, p_{2}\right)=0$ and $y\left(p_{0}, p_{1}, p_{2}\right)=0$. If $L=(z=0) \cap(w=0)$, then $z\left(p_{0}, p_{1}, p_{2}\right)=0$ and $w\left(p_{0}, p_{1}, p_{2}\right)=0$. Thus, these parameter values correspond to base points $\mathrm{p}$ of the parameterization. The multiplicity of this factor $p_{0} u_{0}+p_{1} u_{1}+p_{2} u_{2}$ in the $u$-resultant is called the order of the base point, denoted $o(\mathbf{p})$.

The remaining factors depend on the coefficients $\lambda_{i}$ and $\delta_{i}$. Since there are only a finite number of base points, $L$ may be chosen so that each of these factors is of the form $q_{0} u_{0}+$ $q_{1} u_{1}+q_{2} u_{2}$ where $\left(q_{0}, q_{1}, q_{2}\right)$ is distinct from any base point. Thus, the image of these remaining points are exactly the points of $L \cap S$.

Theorem 2 If $S$ is rational surface with a parameterization of degree d, then the implicit degree of $S$ is exactly $d^{2}-\sum_{j} o\left(\mathbf{p}_{j}\right)$ where $\mathbf{p}_{j}$ denote the base points of the parameterization.

The main objective of this paper is to establish a tighter bound on the implicit degree of the polygonal patches used in [War92]. Such a bound is useful for several reasons. The complexity of many geometric applications for processing such surface patches depends on the implicit degree of the patch. For example, the implicit degree bounds the number of intersections of a straight line and the patch. This quantity directly affects the time required 
for ray tracing the surface patch. The degree of an intersection curve between two such patches is bounded by the product of the degrees of the patches. Bounding the implicit degree of $S$ is also an important first step in the process of creating specialized resultants for converting $S$ to its implicit form.

\section{$3 \quad$ Intersection multiplicity and Newton polygons}

The key to establishing a bound on the degree of a polygonal Bézier patch is the relationship between the orders of the base points of $(f=0) \cap(g=0)$ and the Newton polygons associated with $f$ and $g$. Recall that the order of a base point $\left(p_{0}, p_{1}, p_{2}\right), o(\mathbf{p})$, is simply the power to which the linear factor $p_{0} u_{0}+p_{1} u_{1}+p_{2} u_{2}$ appears in the $u$-resultant when the coefficients $\lambda_{i}$ and $\delta_{i}$ that determine the line $L$ are treated as indeterminates. A base point $\mathbf{p}$ of order greater than one corresponds to several intersection points of $f=0$ and $g=0$ all lying at $\mathbf{p}$.

Intersection multiplicity is a tool for precisely measuring the number of intersection points that coincide at $\mathbf{p}$. Intersection multiplicity was originally developed to allow strict equality in Bézoutś theorem. [Ful78] shows that any measure satisfying the following seven axioms is equivalent to the notion of intersection multiplicity from Bézoutś theorem.

1. $I_{\mathrm{p}}(f, g) \geq 0$ and $I_{\mathrm{p}}(f, g)=\infty$ if $f=0$ and $g=0$ share a common curve at $p$.

2. $I_{\mathbf{p}}(f, g)=0$ if and only if $f(\mathbf{p}) \neq 0$ or $g(\mathbf{p}) \neq 0$.

3. $I_{\mathbf{p}}(f, g)=1$ if and only if $f=0$ and $g=0$ have distinct tangent lines at $p$.

4. $I_{\mathbf{p}}(f, g)=I_{\mathbf{p}}(g, f)$.

5. $I_{\mathrm{p}}(f, g h)=I_{\mathbf{p}}(f, g)+I_{\mathbf{p}}(f, h)$.

6. $I_{\mathbf{p}}(f, g)=I_{\mathbf{p}}(f, a f+g)$ for all $a$ in $\mathbf{C}\left[\alpha_{0}, \alpha_{1}, \alpha_{2}\right]$.

7. $I_{\mathbf{p}}(f, g)$ is invariant under an affine change of coordinates.

For a fixed choice of $L$, we claim that the multiplicity of a linear factor $p_{0} u_{0}+p_{1} u_{1}+p_{2} u_{2}$ in the $u$-resultant of $f$ and $g$ is exactly the intersection multiplicity of $f=0$ and $g=0$ at $\mathbf{p}=\left(p_{0}, p_{1}, p_{2}\right)$. With $o(\mathbf{p})$ so defined, it is a simple exercise to show that $o(\mathbf{p})$ satisfies these seven axioms. 
Given this equivalence, we next show that the intersection multiplicity of two curves $f=0$ and $g=0$ at the origin is related to the shape of the Newton polygons for their defining polynomials. If $f$ is written as the sum of monomials,

$$
f(s, t)=\sum_{i j} f_{i j} s^{i} t^{j}
$$

the Newton polygon $P$ of $f$ is defined as the convex hull of $\left\{(i, j) \mid f_{i j} \neq 0\right\}$. If $f$ is not divisible by $s$ or $t$, then $P$ touches both the $s$ and $t$-axes. The complement of $P$ with respect to the quadrant $(s \geq 0) \cap(t \geq 0)$ forms two regions. The bounded region $R$ containing the origin is called the order polygon of $f$. Figure 2 shows the an example of the Newton polygon and the order polygon for a polynomial.

Theorem 3 Let $f(s, t)$ and $g(s, t)$ be two polynomials of degree $d$ sharing the same order polygon $R$. Then, the intersection multiplicity of $f=0$ and $g=0$ at the origin, $I_{0}(f, g)$, is greater than or equal to twice the area of $R$.

Proof: We will show that the bound holds for $f$ and $g$ with symbolic coefficients. Therefore, the bound must also hold for any specialization of the coefficients.

Let

$$
\begin{aligned}
& f(s, t)=\sum_{i=0}^{d} f_{i}(s) t^{i}, \\
& g(s, t)=\sum_{i=0}^{d} g_{i}(s) t^{i} .
\end{aligned}
$$

The Sylvester matrix $M$ for $f$ and $g$ with respect to $t$ is a $2 d$ by $2 d$ matrix of the form:

$$
\left(\begin{array}{cccccccc}
f_{0} & f_{1} & \ldots & f_{d} & 0 & \ldots & \ldots & 0 \\
0 & f_{0} & f_{1} & \ldots & f_{d} & 0 & \ldots & 0 \\
\ldots & \ldots & \ldots & \ldots & \ldots & \ldots & \ldots & \ldots \\
0 & \ldots & \ldots & 0 & f_{0} & f_{1} & \ldots & f_{d} \\
g_{0} & g_{1} & \ldots & g_{d} & 0 & \ldots & \ldots & 0 \\
0 & g_{0} & g_{1} & \ldots & g_{d} & 0 & \ldots & 0 \\
\ldots & \ldots & \ldots & \ldots & \ldots & \ldots & \ldots & \ldots \\
0 & \ldots & \ldots & 0 & g_{0} & g_{1} & \ldots & g_{d}
\end{array}\right)
$$

Figure 2: The Newton polygon and order polygon for a polynomial 
where the $f_{i}$ 's appear in $d$ rows and the $g_{i}$ 's appear in $d$ rows. The resultant $\operatorname{Res}(f, g, t)$ of $f$ and $g$ with respect to $t$ is the the determininant of the Sylvester matrix [vdW49]. Since $f$ and $g$ have symbolic coefficients, all intersection points of $f=0$ and $g=0$ have distinct $s$ coordinates. Therefore, using Fulton's axiomatic characterization of intersection multiplicity, it is possible to show that $I_{0}(f, g)$ is the maximum $k$ such that $s^{k}$ divides $\operatorname{Res}(f, g, t)$.

We now show that $s^{2 * a r e a(R)}$ divides $\operatorname{Res}(f, g, t)$. If $R$ lies in the $u v$ plane, let $\left(\hat{r}_{m}, m\right)$ be the coordinates of $R \cap(v=m)$ with greatest $u$ coordinate. Let $r_{m}=\left\lceil\hat{r}_{m}\right\rceil$. Since $R$ is the order polygon for $f$ and $g, s^{r_{m}}$ divides both $f_{m}$ and $g_{m}$.

Next, we transform $M$ as follows. If $1 \leq m \leq d$, we multiply row $m$ of $M$ by $s^{r_{m}}$. For $d<m \leq 2 d$, we multiply row $m$ of $M$ by $s^{r_{m}-d}$. This result is the new matrix $\hat{M}$.

$$
\left(\begin{array}{cccccccc}
s^{r_{1}} f_{0} & s^{r_{1}} f_{1} & \ldots & s^{r_{1}} f_{d} & 0 & \ldots & \ldots & 0 \\
0 & s^{r_{2}} f_{0} & s^{r_{2}} f_{1} & \ldots & s^{r_{2}} f_{d} & 0 & \ldots & 0 \\
\ldots & \ldots & \ldots & \ldots & \ldots & \ldots & \ldots & \ldots \\
0 & \ldots & \ldots & 0 & s^{r_{d}} f_{0} & s^{r_{d}} f_{1} & \ldots & s^{r_{d}} f_{d} \\
s^{r_{1}} g_{0} & s^{r_{1}} g_{1} & \ldots & s^{r_{1}} g_{d} & 0 & \ldots & \ldots & 0 \\
0 & s^{r_{2}} g_{0} & s^{r_{2}} g_{1} & \ldots & s^{r_{2}} g_{d} & 0 & \ldots & 0 \\
\ldots & \ldots & \ldots & \ldots & \ldots & \ldots & \ldots & \ldots \\
0 & \ldots & \ldots & 0 & s^{r_{d}} g_{0} & s^{r_{d}} g_{1} & \ldots & s^{r_{d}} g_{d}
\end{array}\right)
$$

The determinant of $\hat{M}$ is $\operatorname{Res}(f, g, t)$ times $s$ to the power

$$
2 * \sum_{i=1}^{d} r_{i} .
$$

Column $n$ of $\hat{M}$ can be divided by some maximal power of $s, s^{c_{n}}$. Since $s^{r_{m}}$ divides $f_{m}$ and $g_{m}$, it is clear from the structure of $\hat{M}$, that the $c_{n}$ 's are related to the $r_{m}$ 's in the following manner:

$$
c_{n} \geq \min _{\substack{i, j, \geq 0 \\ i+j=n}} r_{i}+r_{j}
$$

To complete the theorem, we must show that

$$
\sum_{n=1}^{2 d} c_{n}-2 * \sum_{i=1}^{d} r_{i} \geq 2 * \operatorname{area}(R) .
$$

Let $\left(u_{0}, v_{0}\right), \ldots,\left(u_{h}, v_{h}\right)$ be the set of points with integral coordinates that lie on the common boundary of $R$ and the Newton polygon for $f$ and $g$ ordered such that $v_{k}<v_{k+1}$. Let $v_{-1}=v_{0}=0$ and $v_{h}=v_{h+1}=d$. Then the bound of equation 4 can be refined using the following lemma. 
Lemma 1 For $v_{k-1}+v_{k} \leq n \leq v_{k}+v_{k+1}$

$$
\min _{\substack{i, j, \geq 0 \\ i+j=n}} r_{i}+r_{j}=r_{v_{k}}+r_{n-v_{k}}
$$

Proof: If $v_{k-1}+v_{k} \leq n \leq 2 * v_{k}$, then $v_{k-1} \leq n-v_{k} \leq v_{k}$. Therefore, $\left(\hat{r}_{v_{k}}, v_{k}\right)$ and $\left(\hat{r}_{n-v_{k}}, n-v_{k}\right)$ lie on a common edge of the Newton polygon. Due to the convexity of the Newton polygon,

$$
\hat{r}_{i}+\hat{r}_{j} \geq \hat{r}_{v_{k}}+\hat{r}_{n-v_{k}}
$$

for all $i+j=n$.

But, $\hat{r}_{v_{k}}=u_{k}=r_{v_{k}}$. Therefore,

$$
r_{i}+r_{j}=\left\lceil\hat{r}_{i}\right\rceil+\left\lceil\hat{r}_{j}\right\rceil \geq\left\lceil\hat{r}_{i}+\hat{r}_{j}\right\rceil \geq\left\lceil\hat{r}_{v_{k}}+\hat{r}_{n-v_{k}}\right\rceil=r_{v_{k}}+\left\lceil\hat{r}_{n-v_{k}}\right\rceil=r_{v_{k}}+r_{n-v_{k}}
$$

for all $i+j=n$. A similar proof holds if $2 * v_{k} \leq n \leq v_{k}+v_{k+1}$.

Applying the lemma to equation 4 , it is possible to show that

$$
\sum_{m=1}^{2 d} c_{m} \geq 2 * \sum_{i=1}^{d} r_{i}+\sum_{k=0}^{h}\left(v_{k+1}-v_{k-1}\right) * r_{v_{k}} .
$$

However, $r_{v_{k}}$ is exactly $u_{k}$. So,

$$
\sum_{m=1}^{2 d} c_{m}-2 * \sum_{i=1}^{d} r_{i} \geq \sum_{k=0}^{h}\left(v_{k+1}-v_{k-1}\right) * u_{k} .
$$

Finally, $\sum_{k=0}^{h}\left(v_{k+1}-v_{k-1}\right) * u_{k}$ is simply two times the area of $R$ expressed as a sequence of trapezoids.

Curves and surfaces defined by polynomials over a sparse set of basis functions are called toric varieties in the algebraic geometry literature [Oda85]. Results relating the number of solutions to a system of equations and the volumes of their Newton polytopes are generally known [Ber75, Kus76]. Specifically, [Oda85, pp.79] gives a bound on intersection multiplicity of several surfaces meeting at a point in terms of Newton polytopes. However, this result is derived in a very general framework that requires a deep understanding of the theory of toric varieties. The purpose of the preceding proof was to establish the theorem in a simple, constructive manner without recourse to the general theory of toric varieties.

The proof of this theorem also suggests a useful algorithm for aiding in the computation of resultants. If $f$ and $g$ share a nontrivial order polygon $R$, then the Sylvester matrix 
for $f$ and $g$ can be preprocessed so as to remove the factor $s^{2 * a r e a(R)}$ always present in the resultant. For example, let

$$
\begin{gathered}
f(s, t)=a s^{2}+b s^{3}+c s t+d s^{2} t+e t^{2}+f s t^{2}+g t^{3}, \\
g(s, t)=A s^{2}+B s^{3}+C s t+D s^{2} t+E t^{2}+F s t^{2}+G t^{3},
\end{gathered}
$$

The order polygon for $f$ and $g$ is the triangle of area two with vertices $(0,0),(2,0)$ and $(0,2)$. The Sylvester matrix for $f$ and $g$ with respect to $t$ is:

$$
\left(\begin{array}{cccccc}
a s^{2}+b s^{3} & c s+d s^{2} & e+f s & g & 0 & 0 \\
0 & a s^{2}+b s^{3} & c s+d s^{2} & e+f s & g & 0 \\
0 & 0 & a s^{2}+b s^{3} & c s+d s^{2} & e+f s & g \\
A s^{2}+B s^{3} & C s+D s^{2} & E+F s & G & 0 & 0 \\
0 & A s^{2}+B s^{3} & C s+D s^{2} & E+F s & G & 0 \\
0 & 0 & A s^{2}+B s^{3} & C s+D s^{2} & E+F s & G
\end{array}\right)
$$

The theorem guarantees that determinant to divisible by $s^{4}$. It is clear that the first column is divisible by $s^{2}$ and the second column is divisible by $s$. However, it is not clear where the final factor of $s$ arises.

The matrix $\hat{M}$ of the proof is achieved by multiplying rows one and four by $s$.

$$
\left(\begin{array}{cccccc}
a s^{3}+b s^{4} & c s^{2}+d s^{3} & e s+f s^{2} & g s & 0 & 0 \\
0 & a s^{2}+b s^{3} & c s+d s^{2} & e+f s & g & 0 \\
0 & 0 & a s^{2}+b s^{3} & c s+d s^{2} & e+f s & g \\
A s^{3}+B s^{4} & C s^{2}+D s^{3} & E s+F s^{2} & G s & 0 & 0 \\
0 & A s^{2}+B s^{3} & C s+D s^{2} & E+F s & G & 0 \\
0 & 0 & A s^{2}+B s^{3} & C s+D s^{2} & E+F s & G
\end{array}\right)
$$

Note that $\operatorname{det}(\hat{M})=s^{2} \operatorname{det}(M)$. Now, column one of $\hat{M}$ is divisible by $s^{3}$, column two by $s^{2}$ and column three by $s$. Dividing out these factors yields a new matrix whose determinant is $\operatorname{det}(M) / s^{4}$.

$$
\left(\begin{array}{cccccc}
a+b s & c+d s & e+f s & g s & 0 & 0 \\
0 & a+b s & c+d s & e+f s & g & 0 \\
0 & 0 & a s+b s^{2} & c s+d s^{2} & e+f s & g \\
A+B s & C+D s & E+F s & G s & 0 & 0 \\
0 & A+B s & C+D s & E+F s & G & 0 \\
0 & 0 & A s+B s^{2} & C s+D s^{2} & E+F s & G
\end{array}\right)
$$


Note that the final matrix has the same zero/nonzero structure as the original Sylvester matrix.

\section{A degree bound for polygonal patches}

Given the theorem of the previous section, we now prove a bound on the degree of a polygonal patch $S$ in terms of the Newton polygon of its weight function. We assume that the Newton polygon touches each side of the of the domain triangle $T$. Otherwise, the parameterization of $S$ can be trivially rewritten as a parameterization of lower degree.

Theorem 4 Let $S$ be a rational Bézier surface defined by a rational parameterization of degree $d$ over a domain triangle $T$. Let $P$ be the Newton polygon for the weight function of $S$. Then the implicit degree of of $S$ is no greater than

$$
\frac{\operatorname{area}(P)}{\operatorname{area}(T)} d^{2}
$$

Proof: Recall from section 2 that the degree of $S$ is defined as the number of intersections of $S$ with a line $L$. If $L$ is defined as the intersection of two planes, then these two planes induce two curves $f=0$ and $g=0$, defined by equations 1 and 2 as functions of $\left(\alpha_{0}, \alpha_{1}, \alpha_{2}\right)$ in the parameter plane.

For any $L$, the polynomials $f$ and $g$ share the same Newton polygon $P$. This polygon partitions the domain triangle $T$ into four parts, $P$ itself and a polygon associated with each vertex of $T$. Let $R$ be the polygonal region associated with vertex (1,0,0) (See figure 3 ).

We first show that $f=0$ and $g=0$ intersect at $(1,0,0)$ with at least multiplicity

$$
\frac{\operatorname{area}(R)}{\operatorname{area}(T)} d^{2}
$$

Let $\bar{f}$ and $\bar{g}$ be the power basis representations of $f$ and $g$ respectively, under the transformation

$$
\begin{aligned}
s & =\frac{\alpha_{1}}{\alpha_{0}} \\
t & =\frac{\alpha_{2}}{\alpha_{0}}
\end{aligned}
$$

Figure 3: Partitioning a domain triangle 
The domain triangle $T$ is transformed to a quadrant in the Cartesian plane. The Bernstein basis function $B_{i_{0} i_{1} i_{2}}$ is transformed into the monomial $\frac{s^{i_{1}} t^{i_{2}}}{1+s+t^{d}}$ times some constant. To complete the transformation, we multiply by $(1+s+t)^{d}$ to clears all denominators. This multiplication does affect the intersection multiplicity of $\bar{f}$ and $\bar{g}$ at the origin since $(1+s+t)$ does not vanish at the origin.

In terms of Newton polygons, the basis function $B_{i_{0} i_{1} i_{2}}$ with index $\left(i_{0} / d, i_{1} / d, i_{2} / d\right)$ is mapped to the monomial $s^{i_{1}} t^{i_{2}}$ with index $\left(i_{1}, i_{2}\right)$. For a general polynomial of degree $d$, an index point $\left(i_{0} / d, i_{1} / d, i_{2} / d\right)$ is mapped to the point $\left(i_{1}, i_{2}\right)$ lying in the triangle with vertices $(0,0),(d, 0)$ and $(0, d)$.

Let $\bar{P}$ be the image of $P$ under this index mapping. Then $\bar{P}$ is the Newton polygon for $\bar{f}$ and $\bar{g}$. Let $\bar{R}$ be the order polygon for $\bar{f}$ and $\bar{g}$. The inverse image of $\bar{R}$ under the index mapping is exactly $R$.

By theorem 3, the intersection multiplicity at the origin is no less than 2 area $(\bar{R})$. However, under the index mapping,

$$
\frac{\operatorname{area}(\bar{R})}{d^{2} / 2}=\frac{\operatorname{area}(R)}{\operatorname{area}(T)}
$$

If the intersection multiplicity of $f$ and $g$ is greater than $\frac{\operatorname{area} R}{\operatorname{area} T} d^{2}$ for all possible $L$, then the order of the base point at $(1,0,0)$ is greater than or equal to this quantity.

Since a similar proof holds for the remaining two vertices, the sum of the orders of the base points at the vertices of $T$ is bounded below by

$$
\frac{\operatorname{area}(T)-\operatorname{area}(P)}{\operatorname{area}(T)} d^{2} \text {. }
$$

Therefore, the degree of $S$ is bounded above by

$$
\frac{\operatorname{area}(P)}{\operatorname{area}(T)} d^{2} \text {. }
$$

We observe that equality holds in equation 5 when the coefficients of $S$ are chosen generically. Strict equality holds in theorem 3 when the nonzero coefficients are chosen to be generic. The only remaining possibility is that $f$ and $g$ may have base points other than those at the vertices of $T$. Let $q$ be such a base point. Without loss of generality, assume the $\alpha_{0}, \alpha_{1}$ coordinates of $q$ are nonzero. Let $\left(i_{0}, i_{1}, 0\right)$ be vertex of $P$ on $\alpha_{2}=0$. Then, the basis function $B_{i_{0} i_{1} 0}$ is nonzero at $q$. If the parametric functions $(x, y, z, w)$ are perturbed to 
be $\left(x+\epsilon_{1} B_{i_{0} i_{1} 0}, y+\epsilon_{2} B_{i_{0} i_{1} 0}, z+\epsilon_{3} B_{i_{0} i_{1} 0}, w+\epsilon_{4} B_{i_{0} i_{1} 0}\right)$, then $q$ is no longer a base point of this parameterization for almost all values of $\left(\epsilon_{1}, \epsilon_{2}, \epsilon_{3}, \epsilon_{4}\right)$. Therefore, if the parameterization for $S$ has generic coefficients, the degree of $S$ must be exactly that of equation 5 .

\section{Acknowledgments}

The author would like to thank Doug Moore for his assistance in proofreading this paper.

\section{References}

[Ber75] D. N. Bernshtein. The number of roots of a system of equations. Functional Analysis and Applications, 9(3):1-4, July-September 1975. In Russian.

[Cle68] A. Clebsch. Ueber die abbildung algebraischer flächen insbesondere der vierten and fünften ordnung. Mathematische Annalen, 1:253-316, 1868. In German.

[Far88] Gerald Farin. Curves and Surfaces for Computer Aided Geometric Design: A Practical Guide. Academic Press Inc., New York, 1988.

[Ful78] W. Fulton. Algebraic Curves. Benjamin/Cummings, 1978.

[Har77] R. Hartshorne. Algebraic Geometry. Springer-Verlag, 1977.

[Kus76] A. G. Kushnirenko. Newton polytopes and the Bézout theorem. Functional Analysis and Applications, 10(3):82-83, July-September 1976. In Russian.

[Oda85] T. Oda. Convex Bodies and Algebraic Geometry. Springer-Verlag, 1985.

$\left[\mathrm{S}^{+} 70\right] \quad$ Virgil Snyder et al. Selected Topics in Algebraic Geometry. Chelsea Publishing Company, Bronx, New York, 1970.

[Sal14] George Salmon. A Treatise on the Analytic Geometry of Three Dimensions. Longmans, Green, London, 1914.

[Sed90] Thomas W. Sederberg. Techniques for cubic algebraic surfaces. IEEE Computer Graphics and Applications, pages 14-25, July 1990.

[vdW49] B. van der Waerden. Modern Algebra I. Frederick Unger, New York, 1949. 
[Wa150] R. Walker. Algebraic Curves. Princeton University, 1950.

[War92] Joe Warren. Creating multisided rational Bézier surfaces using base points. ACM Transactions on Graphics, 11(2):127-139, April 1992. 